\title{
A Closed-Loop Reciprocity Calibration Method for Massive MIMO in Terrestrial Broadcasting Systems
}

\author{
Hua Luo, Yue Zhang, Li-Ke Huang, John Cosmas, Senior Member, IEEE, and Amar Aggoun
}

\begin{abstract}
Massive multi-input multioutput (MIMO) is believed to be an effective technique for future terrestrial broadcasting systems. Reciprocity calibration is one of the major practical challenges for massive MIMO systems operating in time-division duplexing mode. A new closed-loop reciprocity calibration method is investigated in this paper which can support online calibration with a higher accuracy compared to the existing methods. In the first part of the proposed method, an optimized relative calibration is introduced using the same structure of traditional relative calibration, but with less impaired hardware in the reference radio chain. In the second part, a test device (TD)-based calibration is proposed which makes online calibration possible. An experiment setup is built for the measurement of the base station hardware impairments and TD-based calibration implementation. Simulation results and the error vector magnitude of $\mathrm{UE}$ received signal after calibration show that the performance of our proposed method is improved significantly compared to the existing relative calibration methods.
\end{abstract}

Index Terms-Terrestrial broadcasting, DVB, massive MIMO, reciprocity calibration, hardware impairments.

\section{INTRODUCTION}

$\mathbf{N}$ OWADAYS, terrestrial broadcasting technologies are facing a challenge of the increasing data rate demand from the users. High data rate content such as HDTV (High Definition TV) and UHDTV (Ultra-High Definition TV) makes the limited wireless bandwidth even more urgent. Besides, the expectation for future digital terrestrial TV broadcasting systems is not only traditional rooftop receivers, but also mobile devices, which explodes the demand for mobile data traffic and drives the research of new digital terrestrial TV technologies [1]. MIMO is a key technology to increase the capacity and system reliability without any additional wireless bandwidth for terrestrial broadcasting systems [2]-[8]. In [2] and [3], the first TV broadcasting system using MIMO technology is introduced which is named DVB-NGH (Digital Video Broadcasting - Next Generation Handheld). Many other standardization forums such as ISDB (Integrated Services Digital Broadcasting), ATSC (Advanced Television Systems

Manuscript received May 26, 2016; revised July 28, 2016; accepted August 9, 2016.

H. Luo, Y. Zhang, and A. Aggoun are with the University of Bedfordshire, Luton, LU1 3JU, U.K. (e-mail: yue_zhang@ieee.org).

L.-K. Huang is with Cobham Wireless, Stevenage, SG1 2AN, U.K.

J. Cosmas is with Brunel University, Uxbridge, UB8 3PH, U.K.

Color versions of one or more of the figures in this paper are available online at http://ieeexplore.ieee.org.

Digital Object Identifier 10.1109/TBC.2016.2606890
Committee), and DVB-T2 (Second Generation Terrestrial) are considering using MIMO technology. According to [2], MIMO can help increase the capacity up to $80 \%$ over SISO with DVB-NGH. However, it cannot meet the expectation for the future broadcasting systems.

Massive MIMO can make a huge increase on system capacity with a much larger number of antennas configured at the base station (BS) [9]. By utilizing spatial multiplexing, a massive MIMO base station can serve multiple users simultaneously through multi-user beamforming (see [10], [11] and references therein). In order to use multi-user beamforming, the BS needs to know the downlink channel state information. One of the conventional ways to get the downlink (DL) channel state information (CSI) is to feed it back from the terminals which is not practical for massive MIMO because the pilots for channel estimation will take too much time and frequency resources [12]. Thanks to channel reciprocity, the DL CSI can be acquired through uplink (UL) channel estimation in TDD mode [13]. However, because of the hardware impairments of the transceivers, the DL and UL channel are not always reciprocal [14], [15], especially when the BS uses a direct conversion transceiver (DCT) and cheap components with aim of reducing hardware costs in massive MIMO systems. Despite the advantages of low-cost, simplicity and flexibility of DCT, some practical problems arise when implementing a direct-conversion transmitter because of the non-linearity of the radio frequency (RF) component, such as in-phase (I) and quadrature $(\mathrm{Q})$ mismatches, clock jitter, power amplifier (PA) distortion and inter-modulation [16]-[22]. The reciprocity of the channel is broken severely by the impairments which makes reciprocity calibration one of the main challenges of massive MIMO systems' implementation.

Several literatures have discussed reciprocity calibration of massive MIMO systems. A relative calibration method was first proposed and implemented in [15]. The hardware impairments are generally equivalent to the frequency response of the hardware. Rogalin et al. [14] studied an over-the-air calibration protocol which can scale well with the network size for distributed massive MIMO. A least square (LS) problem was generated for reciprocity calibration which took the noise effect into account. In [23], a hardware based calibration setup, which can support online calibration, was studied. However, additional hardware-based circuitries are needed which will increase the cost of BS. All the above methods are all based on the "relatively accurate estimation", in which one of the antennas is used as a reference for calibration. Although this will not result in beam pattern distortion as mentioned in [15], 


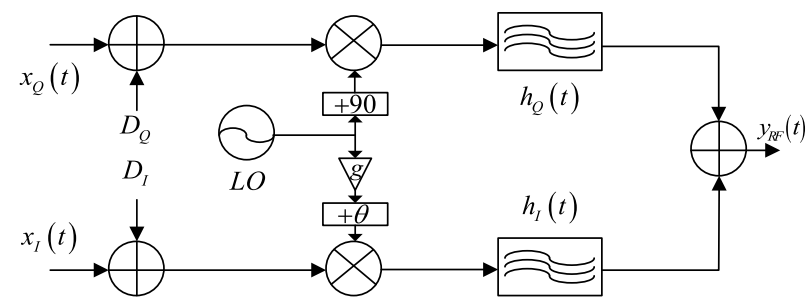

Fig. 1. Hardware imbalance model.

it will degrade the performance (such as EVM) of the base station. A calibration method based on parameter estimation was carried out in [24] which can be performed without any aid of users or external devices. For each pair of transmit (TX) and receive (RX) hardware chain, a parameter was estimated as a function of analog transmit and receive frontends. However, the calibration accuracy is limited because the impairments' parameters of the TX and RX are not estimated separately.

In this work, we propose a closed-loop reciprocity calibration method which can increase the system EVM performance for massive MIMO systems. Zero forcing (ZF) is used as the precoding method. Our major contribution is reciprocity calibration method with higher calibration accuracy compared to the existing methods. Meanwhile, it has the ability to support online calibration without any additional hardware circuit in the BS. The calibration method includes two parts. In the first part, offline-over-cable calibration is performed. The calibration algorithm in this step is the same as the traditional relative calibration but the reference TX/RX hardware chain is specified to be high accuracy. In the second part, a test device (TD) based over-the-air calibration is introduced to support online calibration and also improve the calibration accuracy compared to the existing calibration methods. Furthermore, with our proposed calibration, the BS does not need any additional hardware circuit which can reduce the cost of the BS compared to that in [23] and [24]. Some other references about over-the-air calibration for the antenna system can be found in [25]-[27]. The impact of DCT hardware impairments on massive MIMO systems is firstly analyzed, in particular, DC offset and frequency dependent (FD) I/Q imbalance. Then based on the analysis of hardware impairments, the signal model and system structure of the proposed reciprocity calibration is studied. Finally, a MIMO experiment system is built for measurement of the hardware impairments as well as the implementation of the proposed calibration method.

The rest of the paper is organized as follows. Firstly, the analysis and modeling of hardware impairments are presented in Section II. Secondly, in Section III, the system structure of our reciprocity calibration method is presented including the offline-over-cable calibration and over-the-air online calibration. We then describe the experiment setup, measurements and simulation results in Section IV. Finally, conclusions are made in Section V.

\section{HARDWARE IMPAirments MODEL}

In this section, the hardware impairments of a single antenna DCT system are modeled first as shown in Fig. 1, which shows the RF band imbalance model directly [19] although there exist some models based on the equivalent baseband imbalance [28]-[30]. The massive MIMO system model with hardware impairments is studied next.

\section{A. Single Antenna DCT Impairments Model}

In ideal DCT system, the RF signal can be modeled as:

$$
\begin{aligned}
y(t) & =\Re\left\{x(t) e^{j 2 \pi f_{c} t}\right\} \\
& =x(t) e^{j 2 \pi f_{c} t}+x^{*}(t) e^{-j 2 \pi f_{c} t},
\end{aligned}
$$

where $x(t)$ stands for the complex baseband signal which is modulated by quadrature modulator and $x(t)=x_{I}(t)+j \cdot x_{Q}(t)$. According to (1), the ideal desired baseband signal is shifted to RF frequency band $f_{c}$ and $-f_{c}$ which is its mirror and there is no overlap between these two frequencies. However, due to the hardware impairments of real components, problematic overlapping exists. Generally, as shown in Fig. 1, the DCT impairments include the following factors:

1. The amplitude and phase difference between the LO branches: $g$ and $Q$.

2. The DC offset of I and Q branch.

3. The baseband gain and phase difference between I and Q branch.

4. The mixer conversion gain and amplifier gain difference between I and Q branch.

Factors 1 and 2 are generally frequency-independent while factors 3 and 4 are frequency-dependent effects.

Apparently, by shifting the baseband frequency response to $\mathrm{RF}$, the baseband impairments can be modeled in RF band. The frequency response of the equivalent RF model of FD impairments can be modeled by $h_{I}(t)$ and $h_{Q}(t)$.

Therefore, the impaired RF signal can be written as:

$$
\begin{aligned}
y_{R F}(t)= & \left(x_{I}(t)+D_{I}\right) \cdot g \cdot \cos \left(2 \pi f_{c}+\theta\right) * h_{I}(t) \\
& +\left(x_{Q}(t)+D_{Q}\right) \cdot \cos \left(2 \pi f_{c}+\pi / 2\right) * h_{Q}(t) \\
= & x(t) e^{j 2 \pi f_{c} t} * g_{1}(t)+x^{*}(t) e^{j 2 \pi f_{c} t} * g_{2}(t) \\
& +x^{*}(t) e^{-j 2 \pi f_{c} t} * g_{1}^{*}(t)+x(t) e^{-j 2 \pi f_{c} t} * g_{2}^{*}(t)+D(t),
\end{aligned}
$$

where

$$
\begin{aligned}
& g_{1}(t)=\frac{g \cdot e^{j \theta} h_{I}(t)+h_{Q}(t)}{2} \\
& g_{2}(t)=\frac{g \cdot e^{j \theta} h_{I}(t)-h_{Q}(t)}{2}
\end{aligned}
$$

and $D(t)$ is caused by DC offset. In (2), “*” stands for convolution and $(\cdot)^{*}$ represents conjugation.

Equation (2) shows that the ideal term $x(t) e^{j 2 \pi f_{c} t}+$ $x^{*}(t) e^{-j 2 \pi f_{c} t}$ is distorted by $g_{1}(t)$ and $D(t) . D(t)$ can be easily cancelled by DC offset calibration [37]. Besides, the desired signal is also impaired by $x^{*}(t) e^{j 2 \pi f_{c} t} * g_{2}(t)$ and $x(t) e^{-j 2 \pi f_{c} t} * g_{2}^{*}(t)$ which can be viewed as a result of the image signal distorted by $g_{2}(t)$. The frequency dependent $g_{1}(t)$ results in the common gain of I and Q branch, while $g_{2}(t)$ gives rise to I/Q imbalance of the system. 


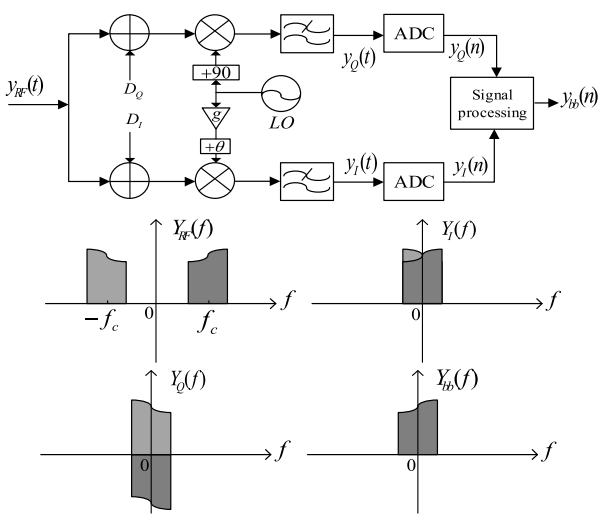

Fig. 2. Demodulation model.

Let $H_{I}(f)$ and $H_{Q}(f)$ be the baseband equivalent of the frequency response of RF imbalanced filter, thus the baseband equivalent model after DC offset calibration can be given as [28]:

$$
Y_{R F}(f)=X(f) G_{1}(f)+X^{*}(-f) G_{2}(f)
$$

where

$$
\begin{aligned}
& G_{1}(f)=g \cdot e^{j \theta} H_{I}(f)+H_{Q}(f) \\
& G_{2}(f)=g \cdot e^{j \theta} H_{I}(f)-H_{Q}(f) .
\end{aligned}
$$

According to (3), the RF signal is distorted both in amplitude and phase. For simplicity, let

$$
\begin{aligned}
Y_{R F}(f) & =M(f) \cdot Y(f) \\
& =|M(f)| \cdot e^{j \phi_{M}(f)} \cdot Y(f),
\end{aligned}
$$

where $M(f)$ stands for the transceiver hardware impairments which are frequency-dependent with the amplitude of $|M(f)|$ and phase of $\phi_{M}(f)$. In particular, $M(f)=T(f)$ or $R(f)$, where $T(f)$ and $R(f)$ stand for the transmitter impairments and receiver impairments respectively. The demodulation model as shown in Fig. 2 shows the conventional I/Q-demodulationbased model from which how the impairments impact the received signal can be seen. In massive MIMO systems with separate local oscillators (LO), each RF chain is independent, so the impairments are independent as well. Here, we only analyze one of the RF chains. In next subsection, the impact of hardware impairments on massive MIMO system will be studied.

\section{B. Massive MIMO Impairments Model}

The massive MIMO system is configured with $N$ antennas in the base station and $K$ user terminals with a single antenna. Through multiuser beamforming, the BS can serve all the terminals simultaneously which helps to achieve high throughput. The downlink baseband OFDM signal model of the $i$ th subcarrier is given by

$$
\mathbf{Y}_{i}=\mathbf{H}_{D, i} \mathbf{X}_{i}+\mathbf{N}_{i}
$$

where $\mathbf{H}_{D, i}=\mathbf{R}_{i, U} \mathbf{B}_{i} \mathbf{T}_{i, B}$ represents the DL channel matrix; $\mathbf{T}_{B, i}=\operatorname{diag}\left(t_{B, i, 1}, \ldots, t_{B, i, N}\right)$ and

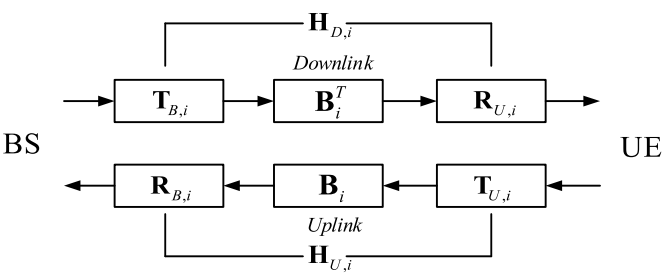

Fig. 3. Reciprocity model.

$\mathbf{R}_{U, i}=\operatorname{diag}\left(r_{U, i, 1}, \ldots, r_{U, i, K}\right)$ denote the BS transmitter frequency-dependent impairments and the terminal receiver impairments respectively; the propagation channel state information is represented by $\mathbf{B}_{i} \in \mathbf{C}^{K \times N} ; \mathbf{N}_{i}$ represents the Gaussian noise vector and $\mathbf{X}_{i}$ is a $N \times 1$ vector of DL precoded symbols at subcarrier $i$. For DCT transceivers, $\mathbf{T}_{B, i}$ can be represented by $T\left(f_{i}\right)$ as described in Section II-A, where $f_{i}$ is the frequency of $i$ th subcarrier.

After precoding, it can be written as [31]

$$
\mathbf{X}_{i}=\mathbf{H}_{U, i}^{H}\left(\mathbf{H}_{U, i} \mathbf{H}_{U, i}^{H}\right)^{-1} \mathbf{D}_{i},
$$

where $\mathbf{H}_{U, i} \in \mathbf{C}^{K \times N}$ is the uplink channel state information obtained by UL CE and $\mathbf{H}_{U, i}^{H}\left(\mathbf{H}_{U, i} \mathbf{H}_{U, i}^{H}\right)^{-1}$ is the ZF precoding matrix, "(.) ${ }^{H}$ " stands for conjugate transpose; $\mathbf{D}_{i}$ denotes the original data symbols at subcarrier $i$.

In order to obtain the UL CSI $\mathbf{H}_{U}$, channel estimation is done by sending pilots from the terminals. The UL pilot signal that is received by the $\mathrm{BS}$ is given by

$$
\mathbf{Y}_{i}^{U}=\mathbf{H}_{U, i} \mathbf{X}_{i}^{P}+\mathbf{N}_{i}^{U}
$$

where $\mathbf{X}_{i}^{P}$ is a $K \times 1$ vector of UL pilot symbols of the $i$ th subcarrier; $\mathbf{H}_{U, i}=\mathbf{R}_{B, i} \mathbf{B}_{i}^{T} \mathbf{T}_{U, i}$ denotes the UL CSI with $\mathbf{T}_{U, i}=$ $\operatorname{diag}\left(t_{U, i, 1}, \ldots, t_{U, i, K}\right)$ and $\mathbf{R}_{B, i}=\operatorname{diag}\left(r_{B, i, 1}, \ldots, r_{B, i, N}\right)$ containing the terminals' transmitter impairments and the BS's receiver impairments respectively; "(. $)^{T}$ " means transpose; $\mathbf{N}_{i}^{U}$ stands for the UL Gaussian noise vector. $\mathbf{R}_{B, i}$ can be represented by $R\left(f_{i}\right)$.

Note that the objective of this paper is to calibrate the nonreciprocity of the BS's UL and DL RF chain. Therefore, only the BS's hardware impairments are considered in our system model so the terminals are assumed to be ideal and they can be treated as perfect transceivers with $\mathbf{R}_{i, U}=\mathbf{I}$ and $\mathbf{T}_{i, U}=\mathbf{I}$. The reciprocity model can be found in Fig. 3. Utilizing some simple algebra, (5) can be rewritten as

$$
\begin{aligned}
\mathbf{Y}_{i} & =\mathbf{H}_{D, i} \mathbf{H}_{U, i}^{H}\left(\mathbf{H}_{U, i} \mathbf{H}_{U, i}^{H}\right)^{-1} \mathbf{D}_{i}+\mathbf{N}_{i} \\
& =\mathbf{B}_{i} \mathbf{T}_{i, B}\left(\mathbf{B}_{i} \mathbf{R}_{i, B}\right)^{H}\left(\mathbf{B}_{i} \mathbf{R}_{i, B}\left(\mathbf{B}_{i} \mathbf{R}_{i, B}\right)^{H}\right)^{-1} \mathbf{D}_{i}+\mathbf{N}_{i} .
\end{aligned}
$$

As shown in (8), it can be easily seen that the symbols received by the terminals are distorted by $\mathbf{T}_{i, B}$ and $\mathbf{R}_{i, B}$. The system performance is degraded by the hardware impairments.

\section{Proposed Calibration Method}

In this section, we present a closed-loop reciprocity calibration method which can support both high accuracy offline and online calibration. The proposed method includes two parts. The first part is based on traditional relative calibration in which a reference antenna is used to receive and transmit pilots 
over cable. We call it optimized relative calibration. In the second part, an innovative test device based online calibration method is studied which can support online calibration and it is named TD based calibration.

\section{A. Optimized Relative Calibration}

In relative calibration, one of the base station antennas is used as a reference antenna. The pilots designed for impairments measurement are transmitted between the other antennas and the reference antenna. The impairments measurement is performed by channel estimation utilizing the known pilots sent out and the received signal by the BS antennas. The channel estimation results from the reference antenna and the other antennas can be written as:

$$
\tilde{\mathbf{H}}_{i, 1 \rightarrow j}=t_{B, i, 1} \mathbf{B}_{i, 1 \rightarrow j} \mathbf{R}_{B, i, j},
$$

where BS antenna 1 is set to be the reference antenna and the other antenna $j$ is between 2 and $N$. Meanwhile, the reverse procedure is carried out and the channel estimates can be given as:

$$
\tilde{\mathbf{H}}_{i, j \rightarrow 1}=\mathbf{T}_{B, i, j} \mathbf{B}_{i, j \rightarrow 1} r_{B, i, 1} .
$$

In particular, the calibration procedure is as follows:

1. Sequentially transmit pilots to and from each base station antenna and reference antenna.

2. Perform channel estimation for the received signal of each transmission.

3. Calculate the calibration coefficients.

After reciprocity calibration, the calibration coefficients are calculated:

$$
\begin{aligned}
\mathbf{C}_{r e l, i} & =\tilde{\mathbf{H}}_{i, j \rightarrow 1}\left(\tilde{\mathbf{H}}_{i, 1 \rightarrow j}\right)^{-1} \\
& =\frac{r_{B, i, 1}}{t_{B, i, 1}} \mathbf{T}_{B, i}\left(\mathbf{R}_{B, i}\right)^{-1} .
\end{aligned}
$$

Using pre-precoding calibration in [11], the UL channel estimation results used for precoding will be

$$
\tilde{\mathbf{H}}_{U, i}=\mathbf{C}_{r e l, i} \mathbf{B}_{i} \mathbf{R}_{B, i} .
$$

By combining (8) and (12), the signal received by the UEs can be rewritten as:

$$
\begin{aligned}
\mathbf{Y}_{i} & =\mathbf{B}_{i} \mathbf{T}_{B, i}\left(\tilde{\mathbf{H}}_{U, i}\right)^{H}\left(\tilde{\mathbf{H}}_{U, i}\left(\tilde{\mathbf{H}}_{U, i}\right)^{H}\right)^{-1} \mathbf{D}_{i}+\mathbf{N}_{i} \\
& =\frac{t_{B, i, 1}}{r_{B, i, 1}} \mathbf{D}_{i}+\mathbf{N}_{i}
\end{aligned}
$$

As shown in (13), the quality of the received signal is affected by the impairments of the reference antenna.

Based on relative calibration, we propose an optimized relative calibration which can improve the calibration accuracy. Simply, some high accuracy components, as well as transceiver structure, are used in the reference antenna chain. Particularly, the LO, mixer, ADC and filter in this RF chain is with high accuracy and the transceiver can be replaced with an intermediate frequency (IF) transceiver. In this case, the impairments of the reference antenna are very small and the calculated reciprocity calibration coefficients are

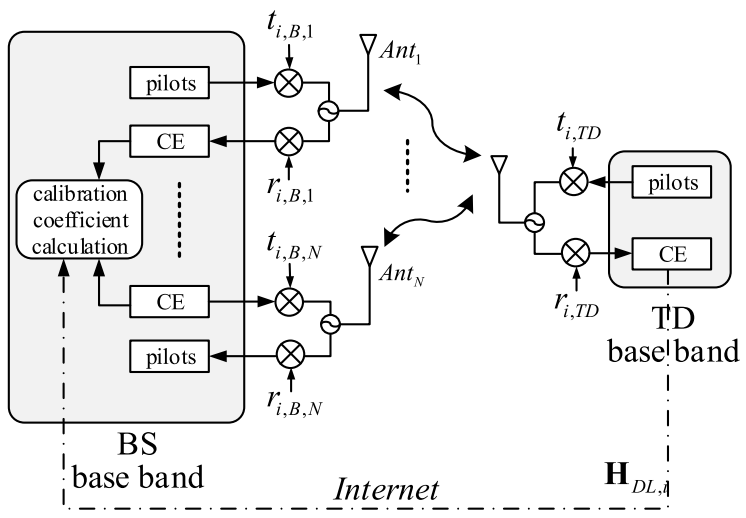

Fig. 4. TD based calibration method.

more accurate compared to the traditional relative calibration coefficients. The UEs received signal will be:

$$
\mathbf{Y}_{i}=\mathbf{D}_{i}+\frac{a_{r e f, T}-a_{r e f, R}}{1+a_{r e f, R}} \mathbf{D}_{i}+\mathbf{N}_{i}
$$

where $a_{r e f, T}$ and $a_{r e f, R}$ stand for the transmitter and receiver impairments of the reference antenna respectively.

\section{B. TD Based Calibration}

Although the BS system is calibrated before release, the accuracy of electronic components naturally drifts over time and it is necessary to calibrate the BS hardware at regular intervals to maintain the intended level of accuracy. By taking advantage of less expensive maintenance of the BS, it is very important that the system can be calibrated online rather than shut down the BS and calibrate it offline.

The TD based calibration that we propose is shown in Fig. 4 which utilizes both UL and DL channel estimation as a closed-loop method. This calibration procedure includes four steps. Firstly, the UL channel estimation is performed by sending pilots from the TD. Secondly, the BS gives a response to the TD and sends pilots back to the TD. Thirdly, the TD transmits the DL channel estimation results $\mathbf{H}_{D L}$ back to the BS. Finally, the calibration coefficient is calculated according to the DL and UL channel estimation results. The calibration is done by multiplying the calibration coefficient with the UL channel estimation results between the BS and UEs.

We understand that the BS may not transmit pilots to the UEs during normal working mode in this scenario. Assume that in this case, the BS can switch to a so-called calibration mode that can response to the TD pilots and send pilots back to TD which makes online calibration possible. As the BS only sends pilots to the TD in calibration mode, the time-frequency resource will not be a problem to orthogonalize the pilots of each antenna element. In order to feed the DL channel estimation results back to $\mathrm{BS}$, the $\mathrm{BS}$ can be typically connected to the wired Internet [14], [32]. Since the wired Internet is used for uploading DL CSI and the BS only responses to the TD uplink pilots for sending calibration pilots via downlink, the Internet latency between the BS and TD will not affect the calibration results. 
After DL and UL channel estimation, the calibration coefficient can be calculated as

$$
\begin{aligned}
\mathbf{C}_{T D, i} & =\mathbf{H}_{D L, i}\left(\mathbf{H}_{U L, i}\right)^{-1} \\
& =\frac{r_{T D, i}}{t_{T D, i}} \mathbf{T}_{B, i}\left(\mathbf{R}_{B, i}\right)^{-1},
\end{aligned}
$$

where $\mathbf{H}_{D L, i}$ and $\mathbf{H}_{U L, i}$ are the DL and UL channel matrix between the BS and TD at subcarrier $i$ respectively; $t_{T D, i}$ and $r_{T D, i}$ are the transmitter and receiver impairments of TD which can be very small if the TD is built with accurate components and sufficiently calibrated in advance. Hence, the impairments of TD are assumed to be approximate to 0 with remaining calibration error $a_{T}$ and $a_{R}$ respectively. Using pre-precoding calibration in [11], the UL channel estimation results used for precoding will be

$$
\tilde{\mathbf{H}}_{U, i}=\mathbf{C}_{T D, i} \mathbf{B}_{i} \mathbf{R}_{B, i} .
$$

Combining (8) and (16), the signal received by the UEs can be rewritten as:

$$
\begin{aligned}
\mathbf{Y}_{i} & =\mathbf{B}_{i} \mathbf{T}_{B, i}\left(\tilde{\mathbf{H}}_{U, i}\right)^{H}\left(\tilde{\mathbf{H}}_{U, i}\left(\tilde{\mathbf{H}}_{U, i}\right)^{H}\right)^{-1} \mathbf{D}_{i}+\mathbf{N}_{i} \\
& =\frac{t_{T D, i}}{r_{T D, i}} \mathbf{D}_{i}+\mathbf{N}_{i} \\
& =\mathbf{D}_{i}+\frac{a_{T}-a_{R}}{1+a_{R}} \mathbf{D}_{i}+\mathbf{N}_{i},
\end{aligned}
$$

which means that the impairments caused by the transceivers of the BS is calibrated with residual error of $\left(a_{T}-a_{R}\right) /\left(1+a_{R}\right)$. In order to maintain the accuracy of calibration as much as possible, the TD is designed to exhibit low hardware impairments and the moving average algorithm can be used for the channel estimates, which can help to reduce the impact of noise. Moreover, some appropriately accurate channel estimation method can be introduced as well [34].

\section{Simulation and Measurement Results}

In this section, the performance of the proposed calibration method is evaluated via MATLAB simulation in which the hardware impairments of BS are modeled by the experimental measurement results. The experiment is divided into two steps. In the first step, a well calibrated TD is used to measure the hardware impairments of the base station and calculate the reciprocity calibration coefficients. Based on the measurement data, the MATLAB simulation for reciprocity calibration is performed. In the second step, the proposed reciprocity calibration method is implemented and the EVM performance is analyzed by processing the received data at the UE side in MATLAB. The configuration of this experiment consists of 8 antennas at the BS and 2 single antenna terminals, which cannot be considered as massive MIMO. Therefore, a computer simulation of our proposed method for massive MIMO systems is introduced as well.

\section{A. Experiment Setup}

This experiment is based on a Linux Server communicating with each National Instrument (NI) Universal Software Radio Peripheral (USRP) (DCT) via Internet and the system

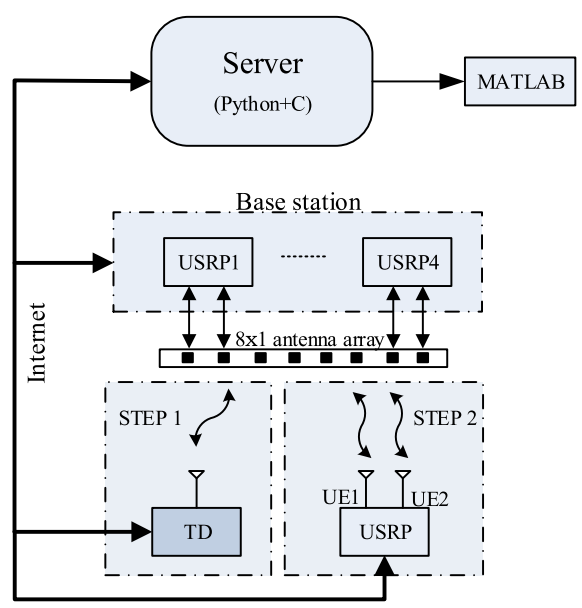

(a)

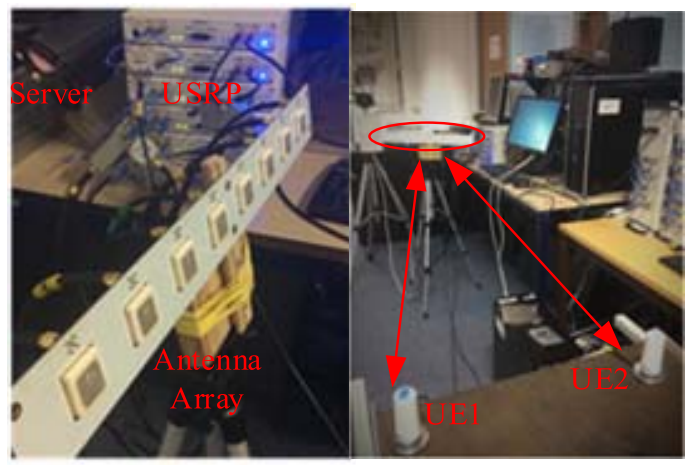

(b)

Fig. 5. Measurement setup. (a) The measurement setup scheme; (b) Scenario of the experiment system implementation.

is controlled to work in TDD mode. We utilize C language to communicate with USRP driver and Python to do some system control, data capture control and graphic user interface etc. As the USRPs are physically separated, time and frequency synchronization need to be carefully designed. All the RF chains are synchronized by sharing a $10 \mathrm{MHz}$ reference signal and a pulse-per-second (PPS) signal from a signal generator between each BS USRP [33].

The experiment in the first step is carried out as shown in Fig. 5(a). The BS is made of four USRP X310 as an 8 antenna transceiver system. An 8 antennas' uniform linear antenna array is allocated as the BS antennas. Each antenna element is omnidirectional and is separated by a half wavelength. The USRP can support $2 \times 2$ MIMO and is configured to work at $2.6 \mathrm{GHz}$ and the bandwidth of USRP is set to be $20 \mathrm{MHz}$. A well calibrated USRP, which has lower DC offset and IQ imbalance compared to the USRPs not well calibrated, is used as TD for the BS hardware impairments measurement. In order to ensure high SNR, the TD is placed within 4 meters distance of the BS in line of sight.

In the second step, the reciprocity calibration is implemented and some modulated data after precoding is sent to the UEs via downlink. A USRP with two different single antennas is used as two UEs as shown in Fig. 5(b). These two 


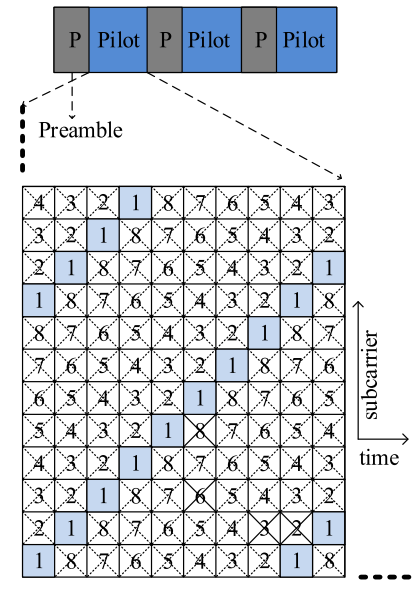

(a)

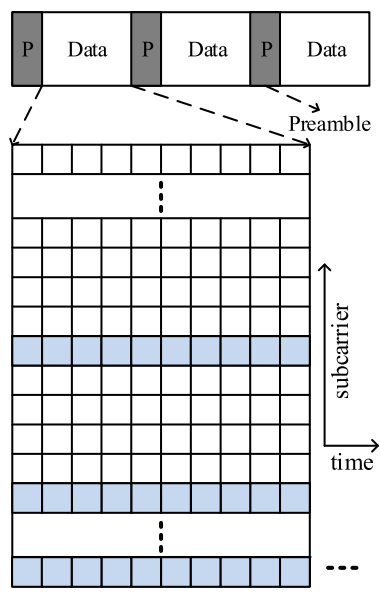

(b)
Fig. 6. Frame structure. (a) The downlink frame and pilot structure for impairments measurement, the blue cell shows the pilot location of antenna 1; (b) the downlink frame and pilot structure for data transmission and $\mathrm{CFO} / \mathrm{SFO}$ cancellation after precoding.

UE antennas are separated by several wavelengths to ensure independence. Precoding at the BS is based on uplink channel estimation results and the original modulated data generated offline. The working procedure in this step is shown as below:

1. UEs send pilots to the BS via uplink.

2. Channel estimation is performed at BS based on the received signal.

3. Calibrate the uplink channel estimates using the reciprocity calibration coefficients in the first step.

4. Precode the modulated data with calibrated uplink channel estimates.

5. BS sends out precoded data.

6. UEs capture data and evaluate the system performance.

The system performance is evaluated via MATLAB by comparing the captured data with the original data bits. In order to reduce the effect of carrier frequency offset (CFO) and sampling frequency offset (SFO), some pilots are inserted into the modulated data. The frame structure and pilots design in this experiment are introduced next.

\section{B. Pilot Design}

Channel estimation is used for hardware impairments' measurement and uplink CSI training in the data transmission step. In this experiment, the pilots' design for channel estimation is different with that for CFO/SFO cancellation.

Firstly, an OFDM frame consists of a preamble and pilot data as shown in Fig. 6(a), which is generated offline. The USRP starts to transmit the OFDM frame repeatedly. The pilot data includes 100 symbols with 1200 subcarriers of each symbol. Each subcarrier bit is set to be 1 or -1 randomly. The pilot of each BS channel is designed by using the comb-type in [12] and is orthogonal in frequency. In each symbol, one subcarrier is only used by one antenna. The pilot of the next symbols is shifted to the next subcarrier so that each antenna uses the full bandwidth through several symbols. As the TD and the
BS are relatively static so the coherent time is long enough for channel estimation.

Moving average is introduced to the channel estimation results in order to reduce the noise effect. After this experiment, the channels between the BS and TD $\left(\mathbf{H}_{D L}\right.$ and $\left.\mathbf{H}_{U L}\right)$ are measured. As the BS and TD are placed in a very short distance and line-of-sight, the channel estimation results are treated as the BS transceivers' impairments.

Secondly, as CFO and SFO are the inherent drawbacks of OFDM-based systems [35], we inserted some block-type pilots in the modulated data for $\mathrm{CFO} / \mathrm{SFO}$ estimation and cancellation in order to reduce the impact on the performance of the proposed calibration method.

In the uplink, the UEs only send pilots up to the BS because only downlink performance is considered in this paper. So the uplink frame structure is the same as the reciprocity calibration frame for channel estimation, which is shown in Fig. 6(a). The downlink data frame contains a preamble and some precoded data as shown in Fig. 6(b). Some pilots are inserted into the data symbols. In order to estimate CFO and SFO, these pilots are precoded using the same precoding matrix as the data. Although inserting pilots into data symbols will cause data rate loss, it will not affect the system EVM performance. The preamble is used for time synchronization and the amplitude of preamble is normalized to 1 as well as the precoded data and the pilots.

\section{Measurement results}

In the first step of our measurement, the DCT hardware impairments are measured via channel estimation as shown in Fig. 8 and Fig. 9. We can see that the DL and UL are not reciprocal and the amplitude of each channel are different. Moreover, the amplitude of impairments is frequencydependent. The impairments we measured includes the fading of the channel over the air and is not equalized.

According to the BS hardware impairments we measured, an $8 \times 2$ MIMO simulation is performed. The propagation channels between the UEs and the BS are configured to be Rayleigh fading. The frequency response of the TD is modeled as i.i.d. complex variables with uniformly distributed phase over $[-\phi, \phi]$ and normalized magnitude in $[1-\sigma, 1+\sigma]$ respectively where $\sigma$ stands for the standard deviation of the squared-magnitude [14] and is measured decibels. Different levels of TD impairments, as well as different SNR, are considered in the simulation.

As shown in Fig. 7, the performance of proposed method is better than that of the hardware based relative calibration method in [23] and the method in [24]. When the impairments of the TD are considered, the EVM of proposed method is larger than the reference, which is with ideal hardware, but still smaller than the existing methods when $\sigma<-20$ $\mathrm{dB}$ and $\phi<2^{\circ}$. Furthermore, the EVM of the received signal is smaller when the residual impairments are smaller. The method in [23] can support online calibration but it is based on relative calibration therefore with less calibration accuracy.

In the second step, the reciprocity calibration is implemented in an 8x2 MIMO system as mentioned 


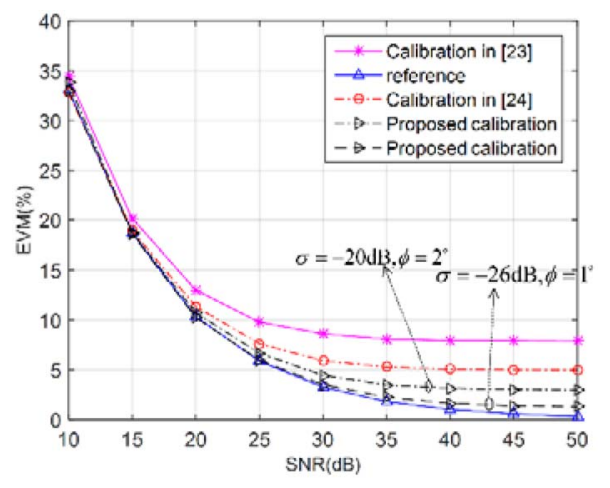

Fig. 7. EVM performance with different SNR.

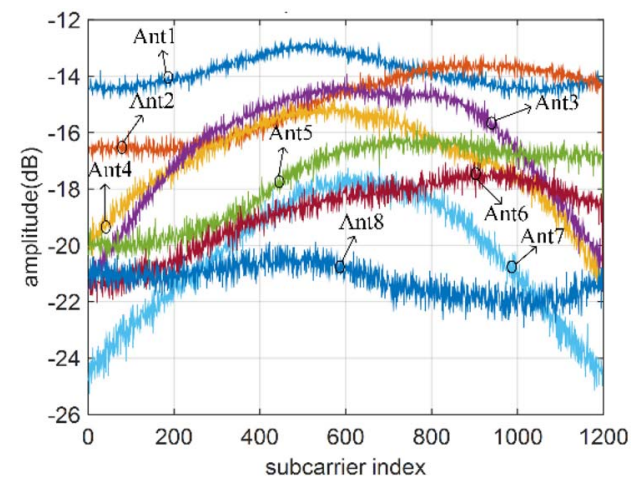

Fig. 8. BS transmitters' amplitude impairments.

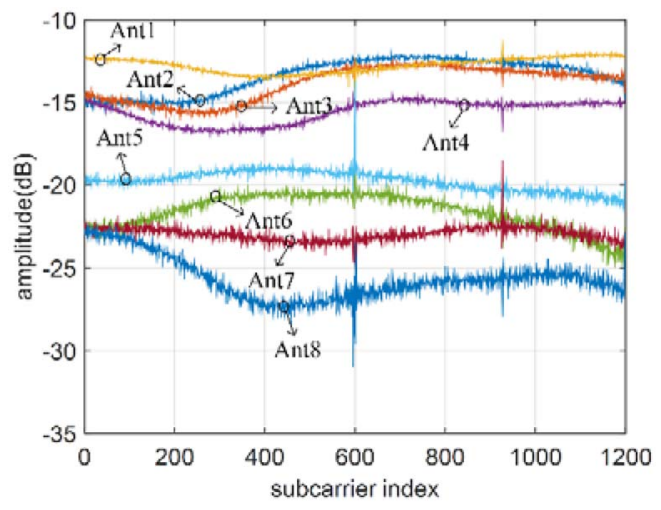

Fig. 9. BS receivers' amplitude impairments.

in Section IV-A. The OFDM data symbols are modulated as 16-QAM (quadrature amplitude modulation). Equalization and $\mathrm{CFO} / \mathrm{SFO}$ cancellation are introduced according to the pilots we insert into the data symbols. The received signal constellation of UE1 with different calibration method is shown in Fig. 10(a) and Fig. 10(b) below. The Root-MeanSquare (RMS) EVM of the former method is $16.34 \%$ and the latter is $5.76 \%$, which means the performance of our proposed calibration method is improved significantly with EVM improvement of around 10.6\% compared to calibration in [23] in this case.

Another simulation is introduced as shown in Fig. 11. Different BS antenna numbers are considered. The received signal SNR is set to be $20 \mathrm{~dB}$. The BS antennas are impaired

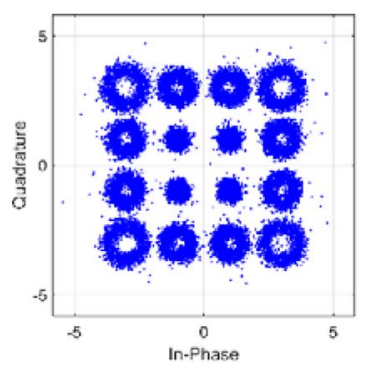

(a)

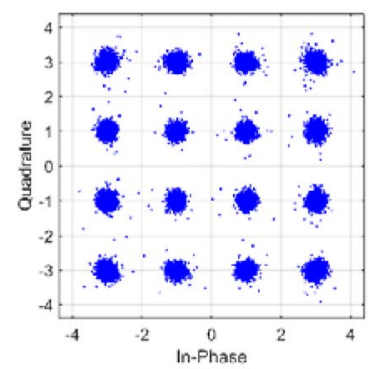

(b)
Fig. 10. Constellation of real captured data. (a) The constellation of received data using traditional relative calibration; (b) the constellation of received data using proposed TD based calibration.

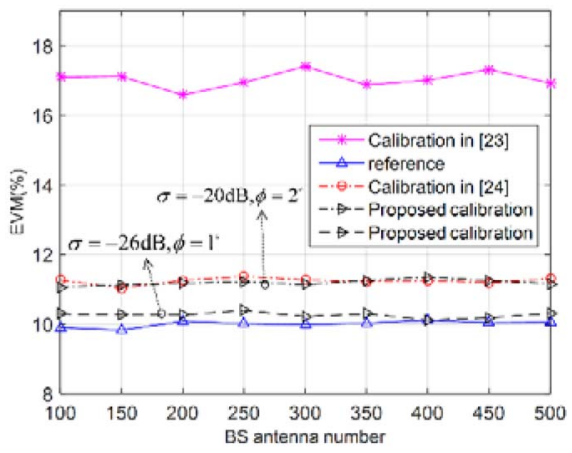

Fig. 11. EVM performance of different BS antenna number.

by $\sigma=-14 \mathrm{~dB}$ and $\phi=10^{\circ}$. We can see that our proposed method is robust to different massive MIMO configurations and the impact of BS hardware impairments cannot be cancelled out by scaling law [36] from reciprocity calibration point of view. The system performance depends on the residual impairments of the TD. When the impairments of the TD are very small, such as $\sigma<-26 \mathrm{~dB}$ and $\phi<1^{\circ}$, the performance of our proposed method is similar and very close to the case that all the BS antennas are ideal but is much smaller than the relative calibration in [23]. When $\sigma=-20 \mathrm{~dB}$ and $\phi=2^{\circ}$, the EVM performance of the proposed method is similar to that in [24].

\section{CONCLUSION}

Reciprocity calibration is one of the major challenges for massive MIMO systems which can be a candidate for future terrestrial broadcasting technologies. This paper proposed a novel and innovative closed-loop reciprocity calibration method for massive MIMO systems with a better performance compared to the existing methods. The proposed method consists of two parts, optimized relative calibration and TD based calibration. The former is performed offline over cable using one of the BS antennas as a reference, which is designed to be high quality and low impairments. The latter is accomplished over air using an accurate specially designed test device. By transmitting the DL CSI from the TD to the BS via the Internet, the calibration coefficient is calculated. In this scenario, the BS can respond to the TD and switch to calibration mode, which makes online calibration possible. 
The reciprocity calibration is performed by multiplying the calibration coefficient with UL channel estimation results between the BS and UEs.

A lab measurement setup is built for BS hardware impairments' measurement and the implementation of TD based calibration. Based on the impairments' measurement, a simulation of different SNR for 8x2 MIMO system is introduced and the comparison between the traditional relative calibration and the optimized relative calibration as well as the TD based calibration is made. The simulation results show that the EVM performance of the proposed calibration method outperforms the existing methods. After that, the TD based calibration is implemented for an $8 \times 2$ MIMO system and the EVM of received signal confirms the validity of our proposed method.

\section{REFERENCES}

[1] D. Vargas, Y. J. D. Kim, J. Bajcsy, D. Gómez-Barquero, and N. Cardona, "A MIMO-channel-precoding scheme for next generation terrestrial broadcast TV systems," IEEE Trans. Broadcast., vol. 61, no. 3, pp. 445-456, Sep. 2015.

[2] D. Vargas, D. Gozalvez, D. Gómez-Barquero, and N. Cardona, "MIMO for DVB-NGH, the next generation mobile TV broadcasting," IEEE Commun. Mag., vol. 51, no. 7, pp. 130-137, Jul. 2013.

[3] D. Gómez-Barquero, C. Douillard, P. Moss, and V. Mignone, "DVB-NGH: The next generation of digital broadcast services to handheld devices," IEEE Trans. Broadcast., vol. 60, no. 2, pp. 246-257, Jun. 2014.

[4] C. Gomez-Calero, L. C. Navarrete, L. de Haro, and R. Martinez, "A $2 \times 2$ MIMO DVB-T2 system: Design, new channel estimation scheme and measurements with polarization diversity," IEEE Trans. Broadcast., vol. 56, no. 2, pp. 184-192, Jun. 2010.

[5] J.-S. Han, J.-S. Baek, and J.-S. Seo, "MIMO-OFDM transceivers with dual-polarized division multiplexing and diversity for multimedia broadcasting services," IEEE Trans. Broadcast., vol. 59, no. 1, pp. 174-182, Mar. 2013.

[6] S. Saito et al., " $8 \mathrm{~K}$ terrestrial transmission field tests using dualpolarized MIMO and higher-order modulation OFDM," IEEE Trans. Broadcast., vol. 62, no. 1, pp. 306-315, Mar. 2016.

[7] J. H. Seo, T. J. Jung, H. M. Kim, and D. S. Han, "Improved polarized $2 \times 2$ MIMO spatial multiplexing method for DVB-NGH system," IEEE Trans. Broadcast., vol. 61, no. 4, pp. 729-733, Dec. 2015.

[8] T. Cheng, K. Peng, F. Yang, J. Song, and Z. Yang, "A near-capacity MIMO coded modulation scheme for digital terrestrial television broadcasting," IEEE Trans. Broadcast., vol. 61, no. 3, pp. 367-375, Sep. 2015.

[9] L. Dai, Z. Wang, and Z. Yang, "Next-generation digital television terrestrial broadcasting systems: Key technologies and research trends," IEEE Commun. Mag., vol. 50, no. 6, pp. 150-158, Jun. 2012.

[10] F. Rusek et al., "Scaling up MIMO: Opportunities and challenges with very large arrays," IEEE Signal Process. Mag., vol. 30, no. 1, pp. 40-60, Jan. 2013.

[11] W. Zhang et al., "Large-scale antenna systems with UL/DL hardware mismatch: Achievable rates analysis and calibration," IEEE Trans. Commun., vol. 63, no. 4, pp. 1216-1229, Apr. 2015.

[12] S. Coleri, M. Ergen, A. Puri, and A. Bahai, "Channel estimation techniques based on pilot arrangement in OFDM systems," IEEE Trans. Broadcast., vol. 48, no. 3, pp. 223-229, Sep. 2002.

[13] G. Thiagarajan and C. R. Murthy, "Novel transmit precoding methods for Rayleigh fading multiuser TDD-MIMO systems with CSIT and no CSIR," IEEE Trans. Veh. Technol., vol. 64, no. 3, pp. 973-984, Mar. 2015.

[14] R. Rogalin et al., "Scalable synchronization and reciprocity calibration for distributed multiuser MIMO," IEEE Trans. Wireless Commun. vol. 13, no. 4, pp. 1815-1831, Apr. 2014.

[15] C. Shepard et al., "Argos: Practical many-antenna base stations," in Proc. 18th Annu. Int. Conf. Mobile Comput. Netw., Istanbul, Turkey, 2012, pp. 53-64.
[16] J. K. Cavers and M. W. Liao, "Adaptive compensation for imbalance and offset losses in direct conversion transceivers," IEEE Trans. Veh. Technol., vol. 42, no. 4, pp. 581-588, Nov. 1993.

[17] A. A. Abidi, "Direct-conversion radio transceivers for digital communications," IEEE J. Solid-State Circuits, vol. 30, no. 12, pp. 1399-1410, Dec. 1995.

[18] B. Debaillie, P. V. Wesemael, G. Vandersteen, and J. Craninckx, "Calibration of direct-conversion transceivers," IEEE J. Sel. Topics Signal Process., vol. 3, no. 3, pp. 488-498, Jun. 2009.

[19] W. Li et al., "Self-IQ-demodulation based compensation scheme of frequency-dependent IQ imbalance for wideband direct-conversion transmitters," IEEE Trans. Broadcast., vol. 61, no. 4, pp. 666-673, Dec. 2015.

[20] F. Gregorio, J. Cousseau, S. Werner, T. Riihonen, and R. Wichman, "EVM analysis for broadband OFDM direct-conversion transmitters," IEEE Trans. Veh. Technol., vol. 62, no. 7, pp. 3443-3451, Sep. 2013.

[21] D. Tandur and M. Moonen, "Joint adaptive compensation of transmitter and receiver IQ imbalance under carrier frequency offset in OFDM-based systems," IEEE Trans. Signal Process., vol. 55, no. 11, pp. 5246-5252, Nov. 2007.

[22] M. Inamori, A. M. Bostamam, Y. Sanada, and H. Minami, "IQ imbalance compensation scheme in the presence of frequency offset and dynamic DC offset for a direct conversion receiver," IEEE Trans. Wireless Commun., vol. 8, no. 5, pp. 2214-2220, May 2009.

[23] M. Petermann et al., "Multi-user pre-processing in multi-antenna OFDM TDD systems with non-reciprocal transceivers," IEEE Trans. Commun., vol. 61, no. 9, pp. 3781-3793, Sep. 2013.

[24] J. Choi, "Downlink multiuser beamforming with compensation of channel reciprocity from RF impairments," IEEE Trans. Commun., vol. 63 , no. 6, pp. 2158-2169, Jun. 2015.

[25] P. Kyösti, T. Jämsä, and J.-P. Nuutinen, "Channel modelling for multiprobe over-the-air MIMO testing," Int. J. Antenna Propag., vol. 2012, Mar. 2012, Art. no. 615954, doi:10.1155/2012/615954.

[26] Y. Jing, Z. Wen, H. Kong, S. Duffy, and M. Rumney, "Two-stage over the air (OTA) test method for MIMO device performance evaluation," in Proc. IEEE Int. Symp. Antennas Propag. (APSURSI), Spokane, WA, USA, Jul. 2011, pp. 71-74.

[27] M. Rumney, R. Pirkl, M. H. Landmann, and D. A. Sanchez-Hernandez, "MIMO over-the-air research, development, and testing," Int. J. Antennas Propag., vol. 2012, May 2012, Art. no. 467695, doi: $10.1155 / 2012 / 467695$.

[28] L. Anttila, M. Valkama, and M. Renfors, "Frequency-selective I/Q mismatch calibration of wideband direct-conversion transmitters," IEEE Trans. Circuits Syst. II, Exp. Briefs, vol. 55, no. 4, pp. 359-363, Apr. 2008

[29] L. Ding, Z. Ma, D. R. Morgan, M. Zierdt, and G. T. Zhou, "Compensation of frequency-dependent gain/phase imbalance in predistortion linearization systems," IEEE Trans. Circuits Syst. I, Reg. Papers, vol. 55, no. 1, pp. 390-397, Feb. 2008

[30] M. Kim, Y. Maruichi, and J.-I. Takada, "Parametric method of frequency-dependent I/Q imbalance compensation for wideband quadrature modulator," IEEE Trans. Microw. Theory Techn., vol. 61, no. 1, pp. 270-280, Jan. 2013.

[31] Q. H. Spencer, C. B. Peel, A. L. Swindlehurst, and M. Haardt, "An introduction to the multi-user MIMO downlink," IEEE Commun. Mag., vol. 42 , no. 10 , pp. 60-67, Oct. 2004.

[32] U. Madhow, Introduction to Communication Systems. Cambridge, U.K.: Cambridge Univ. Press, Nov. 2014, p. 12.

[33] D. Hall, A. Hinde, and Y. Jia, "Multi-channel phase-coherent RF measurement system architectures and performance considerations," in Proc. IEEE Mil. Commun. Conf. (MILCOM), Baltimore, MD, USA, Oct. 2014, pp. $1318-1323$

[34] C. Shin, R. W. Heath, and E. J. Powers, "Blind channel estimation for MIMO-OFDM systems," IEEE Trans. Veh. Technol., vol. 56, no. 2, pp. 670-685, Mar. 2007.

[35] H. Nguyen-Le, T. Le-Ngoc, and C. C. Ko, "RLS-based joint estimation and tracking of channel response, sampling, and carrier frequency offsets for OFDM," IEEE Trans. Broadcast., vol. 55, no. 1, pp. 84-94, Mar. 2009.

[36] E. Björnson, M. Matthaiou, and M. Debbah, "Massive MIMO with non-ideal arbitrary arrays: Hardware scaling laws and circuit-aware design," IEEE Trans. Wireless Commun., vol. 14, no. 8, pp. 4353-4368, Aug. 2015.

[37] A. Loke and F. Ali, "Direct conversion radio for digital mobile phonesdesign issues, status, and trends," IEEE Trans. Microw. Theory Techn., vol. 50, no. 11, pp. 2422-2435, Nov. 2002. 


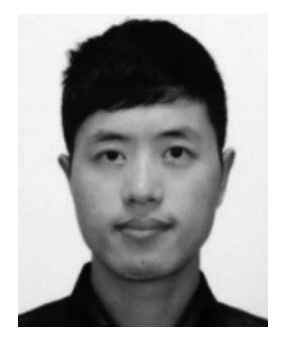

Hua Luo received the B.E. and M.E. degrees from the University of Electronic Science and Technology of China in 2011 and 2014, respectively. He is currently pursuing the Ph.D. degree with the University of Bedfordshire, U.K. His research interests include hardware impairments calibration of massive MIMO systems, MIMO signal processing, MIMO-OFDM systems, and beamforming techniques.

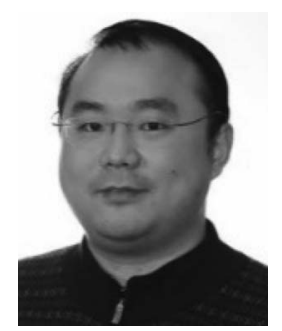

Yue Zhang received the B.E. and M.E. degrees from the Beijing University of Post and Telecommunications, China, in 2001 and 2004, respectively, and the Ph.D. degree from Brunel University, U.K., in 2008, where he was also a Research Engineer for the EU IST FP6 project-PLUTO. Since 2008, he has been a Signal Processing Design Engineer in Microwave Measurement Division, Europe, Anritsu Corporation. He was responsible for the RF/IF, digital, and DSP design for the measurement instruments for various wireless and broadcasting systems. In 2010, he joined the Department of Computer Science and Technology, University of Bedfordshire, Luton, U.K., as a Reader in Signal Processing. He was with Royal Academy of Engineering, U.K., Industrial Secondment with Aeroflex Ltd. His research interests are signal processing, wireless and broadcasting systems, MIMO-OFDM systems, radio propagation model, and multimedia and wireless networks. He currently serves as an Associate Editor for the IEEE Transactions on Broadcasting.

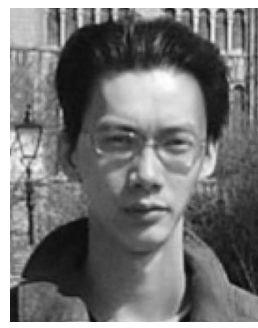

Li-Ke Huang received the B.Sc. degree in electronic engineering from Shenzhen University, China, in 1998, and the Ph.D. degree in communication and signal processing from Imperial College London, U.K., in 2003. He is the Research and Technology Director with Cobham Wireless and a Visiting Professor with the 5G Innovation Centre, University of Surrey. His research interests are in test and measurement technologies for wireless system and in transceiver algorithms and wireless architecture designs for all major communication standards. He is responsible for the new product and core technology innovations contributing to the company's technological and business strategies. He specializes in early technology research and development programs for wireless and digital products, which are often first to the fast moving for 3G, LTE, 4G, and 5G wireless mobile technology markets.

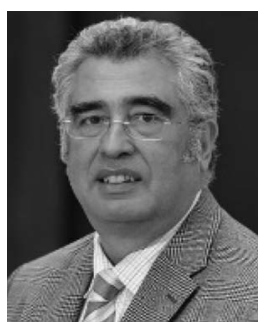

John Cosmas (M'89-SM'08) was born in Colombo, Sri Lanka, in 1956. He received the B.Eng. degree in electronic engineering from Liverpool University in 1978 and the Ph.D. degree from Imperial College London in 1986. He is currently a Professor of Multimedia Systems with the College of Engineering, Design and Physical Sciences, Brunel University London. He co-leads the Wireless Networks and Communications Research Centre and the Course Director of M.Sc. Advanced Multimedia Design and 3-D Technologies. His research interests are concerned with the development of multimedia systems applied to future of broadcasting, cellular communications, 2-D/3-D digital video/graphics media, and the synergies between these technologies toward their application toward the benefit of the environment, health, and societies. He has participated in 11 EU-IST and two EPSRC funded research projects since 1986 and he has led three of these (CISMUNDUS, PLUTO, and 3D MURALE). His latest research is concerned with management of heterogeneous cellular networks, convergence of cellular and ad-hoc networks, 3D MIMO, and efficient software defined networks architectures. He is an Associate Editor of the IEEE Transactions on Broadcasting.

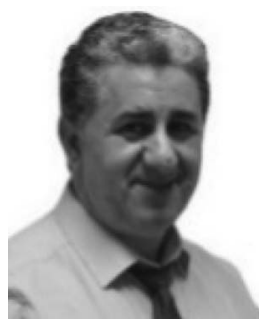

Amar Aggoun received the Ingenieur d'état degree in electronics engineering from École Nationale Polytechnique d'Alger, Algiers, Algeria, and the $\mathrm{Ph} . \mathrm{D}$. degree in electronic engineering from the University of Nottingham. From 1991 to 2013, he held various academic positions with the University of Nottingham, De Montfort University, and Brunel University. In 2013, he joined the University of Bedfordshire as the Head of Computer Science and Technology. His research interests include light-field imaging systems, computer generation and live capture of 3-D integral images, depth measurement, and volumetric data reconstruction, 3-D medical visualization, 3-D video coding, computer vision systems, and real-time digital image/video processing. 